\title{
Crack initiation in viscoelastic materials
}

\author{
Yuval Mulla ${ }^{1}$, Giorgio Oliveri $^{2}$, Johannes T.B. Overvelde ${ }^{2}$, Gijsje H. Koenderink ${ }^{1}{ }^{*}$ \\ ${ }^{1}$ Living Matter Department, AMOLF, Science Park 104, 1098 XG Amsterdam and \\ ${ }^{2}$ Designer Matter Department, AMOLF, Science Park 104, 1098 XG Amsterdam
}

\begin{abstract}
In viscoelastic materials, individually short-lived bonds collectively result in a mechanical resistance which is long-lived but finite, as ultimately cracks appear. Here we provide a microscopic mechanism by which cracks emerge from the nonlinear local bond dynamics. This mechanism is different from crack initiation in solids, which is governed by a competition between elastic and adhesion energy. We provide and numerically verify analytical equations for the dependence of the critical crack length on the bond kinetics and applied stress.
\end{abstract}

Liquids cannot fracture, but solids can. We consider the intermediate case: viscoelastic materials. These materials are made of filaments or particles interconnected by short-lived bonds. This design theme of transient networks is commonly used in both natural and man-made materials such as cytoskeletal polymer networks in cells 1], physical gels [2], associative and telechelic polymers [3, and colloidal gels [4.

The molecular dynamics of transient networks lead to interesting macroscopic mechanics: at times shorter than the bond lifetime the material behaves like a solid [1, while on longer time scales the bonds reorganize and the material deforms viscoelastically [1, 5, 6]. As a result, transient networks are much more deformable than permanent networks [7]. However, viscoelastic materials can resist mechanical stress only for a limited time, after which the system suddenly loses its mechanical percolation, a process which is known as fracturing [3, 8[10. This raises the question how we can design transient networks such that the robustness against stress is optimized, which requires an understanding of the mechanism by which transient networks fracture.

Fracturing of viscoelastic materials is often explained by the Griffith theory of crack initiation in brittle solids 3, 8, 11-14. The Griffith theory predicts that beyond a critical stress, initial defects will grow into macroscopic cracks as the elastic energy released by the crack dominates the surface energy required for separation [15. However, this framework was originally developed for solids, and assumes defects are either static or growing. This assumption is clearly invalid for viscoelastic materials, as defects are not static entities but instead continuously appear and heal [16]. Therefore, viscoelastic materials require a framework which takes into account the reversible bond dynamics.

The seminal work of Bell on cellular adhesion provides such a framework of reversible bond dynamics under force [17], and has received considerable attention in studying small-scale systems such as protein clusters which provide cellular adhesion [18, 19], fracturing of a single colloidal strand [9] and protein unfolding [20].

\footnotetext{
* Corresponding author: g.koenderink@amolf.nl
}

In all of these works, force is assumed to be homogeneously distributed across all bonds, which appears to be a realistic assumption for microscopically small systems. Contrarily, in the context of viscoelastic materials, theoretical [21, 22] and experimental work on both synthetic gels [23] and biopolymer networks [24] has revealed nonaffine deformations upon application of global stress [25]. Indeed, imaging of various networks under stress showed inhomogeneities of the local force which are strongly correlated in space [26 28]. These inhomogeneities might be negligible when considering bulk properties such as the average bond lifetime under stress [29, 30, but likely play a key role in crack initiation. In situ imaging of stressed viscoelastic materials revealed that fracturing occurs via well-defined cracks [3, 11, 31, 32, rather than via the homogeneous degradation expected from the Bell model [17-20, 29, 30, 33, suggesting local rather than global load sharing. At which length scale does the global load sharing assumption become inaccurate? And what determines the fracturing properties of a system of reversible bonds under load beyond this length scale?

To answer these questions we developed a minimal model that includes reversible bond dynamics (figure 1a) in the simplest possible 'material' that is capable of exhibiting spatial inhomogeneity required for studying crack initiation: bonds distributed over a 1D-space, subject to mechanical stress. To account for inhomogeneous load sharing, we assume a force distribution that depends on the local bond spacing (figure 1p). We show that this minimal model system exhibits spontaneous crack initiation and subsequent fracture, in a manner that is consistent with experimental observations in wide range of viscoelastic materials [3, 11, 31, 32]. We verify our results by comparison with a mechanical model. We study the process of crack initiation in more detail by locally 'ablating' bonds (figure 1 1 ), which reveals a critical crack length beyond which fracturing occurs. We provide analytical equations describing the process of crack initiation on basis of the nonlinear bond dynamics, and predict the dependence of the critical crack length on both bond properties and applied stress. Our work reveals that the process of crack initiation in viscoelastic materials is fundamentally different from that in traditional solids, as a consequence of the reversible bond dynamics.

We initialize a one-dimensional (1D) network with $N$ 
equally spaced binding sites using periodic boundary conditions, each bond having a probability $K$ to start in a closed state. Next we model the dynamics of the bonds with a kinetic Monte Carlo scheme [34] using the following bond dynamics:

$$
K=\frac{k_{\text {on }}}{k_{\text {on }}+k_{\text {off }, 0}}
$$

where $k_{\text {on }}$ is the rate of bond closing and $k_{\text {off }, 0}$ the rate of bond opening in the absence of force (figure 1 a). We normalize time by the on-rate, $k_{\text {on }}$. The off-rate increases exponentially with the applied force $f$ on the bond in keeping with the Bell model [17]:

$$
k_{\text {off }}\left(f_{i}\right)=k_{\text {off }, 0} \cdot \exp \left(\frac{f_{i}}{f_{1 / \mathrm{e}}}\right)
$$

where $f_{1 / \mathrm{e}}$ is the force where the off-rate has fallen to $1 / \mathrm{e}$ of $k_{\text {off, } 0}$. We calculate the force per bond $f_{i}$ via

$$
f_{i}=\alpha_{i} \cdot \sigma
$$

where $\sigma$ is the stress on the system and $\alpha$ is a yet to be defined stress intensity factor per bond. In global load sharing, the applied stress is equally divided over all bonds. To investigate the effect of inhomogeneous force distribution as present in any network under stress [26[28, we investigate a local load sharing model. In this model, we assume that the force distribution is dependent on the distance $l_{i}$ of a bond to its nearest neighbor on both sides (figure 1p). Explicitly, we define a stress intensity factor $\alpha$ on a closed bond at site $i$ by:

$$
\alpha_{i}= \begin{cases}N \cdot \frac{l_{i}}{\Sigma_{i} l_{i}} & \text { Local } \\ \frac{N}{\Sigma_{i} n_{i}} & \text { Global }\end{cases}
$$

where $n_{i}$ equals 1 when the bond is closed and 0 when the bond is open. Note that in both modes of load sharing the total amount of force is independent of the bound fraction and normalized by the system size, $\frac{\sum_{i} f_{i}}{N}=\sigma$. We normalize the applied stress by the bond force sensitivity $f_{1 / \mathrm{e}}$. After calculating the force on all bonds, we employ a kinetic Monte Carlo step to either open or close a bond stochastically. We repeat this process of stochastic bond removal/addition until all bonds are removed.

As shown in figure $2 a$, the fraction of closed bonds fluctuates over time, until it drops precipitously to zero at a certain moment that we denote as the rupture time. The rupture time is exponentially distributed - indicative of the stochastic nature of fracturing [35. To test the sensitivity of the average rupture time to the applied stress, we perform simulations at a fixed network size and fixed bond affinity for different levels of stress $(N=20, K=0.9, \sigma=0.5 \ldots 2)$. For both local and global

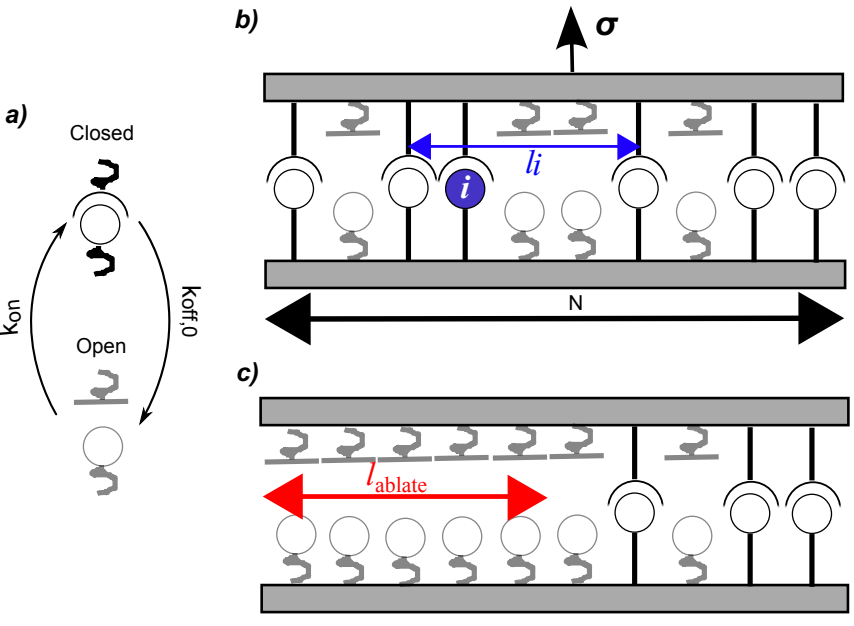

FIG. 1. Schematic of model a) Bonds switch from an open to a closed state with rate $k_{\mathrm{on}}$ and reverse with a rate of $k_{\mathrm{off}, 0}$ in the absence of force. b) Bonds in a closed state share an applied load $\sigma$, where the load distribution depends on the distance $l_{i}$ from bond $i$ to its nearest neighbors. c) We perform bond ablation experiments by opening all bonds in $l_{\text {ablate }}$ adjacent positions to investigate the critical length required for triggering fracturing. Periodic boundary conditions are used to prevent edge effects from influencing the results.

load sharing, we find that the average rupture time shows two distinct regimes with a transition around $<t_{\text {rupt }}>\approx 1$ (figure 2b). As we will explain later on, these regimes correspond to a metastable network at low stress and an unstable network at high stress. Importantly, networks with local load sharing are markedly less robust than globally load sharing networks, with smaller average rupture times at all stresses.

To test how the system size influences the average rupture time, we perform simulations for networks with $N$ varying between 5 and 100 (figure 2k). In case of global load sharing, we see that the average rupture time monotonically increases with system size, as the relative fluctuations of the fraction of closed bonds $\left(\frac{\Sigma_{i} n_{i}(t)}{N}\right)$ decreases [9. 17 19]. In case of local load sharing, we find similar rupture times as compared to globally load sharing networks for small $N$. But strikingly, beyond a critical length (around $N=12$ for these conditions), we find that only in case of local load sharing the rupture time decreases with increasing system size, according to $<t_{\text {rupt }}>\sim N^{-1}$ (inset of figure 22). This dependence suggests a constant crack initiation rate for every 12 bonds at this particular stress. Indeed, kymographs of simulations using local load sharing reveal that fracturing proceeds via cracks rather than homogeneous degradation (figure $2 \mathrm{~d})$.

To understand what sets this critical length for crack initiation, we performed 'ablation experiments' (figure 1.): first we equilibrate the network under stress, next we remove all bonds in $l_{\text {ablate }}$ adjacent positions, then we study whether bond ablation triggered network fracturing. We chose the system size $\mathrm{N}=l_{\text {ablate }} \cdot 10$, such that 

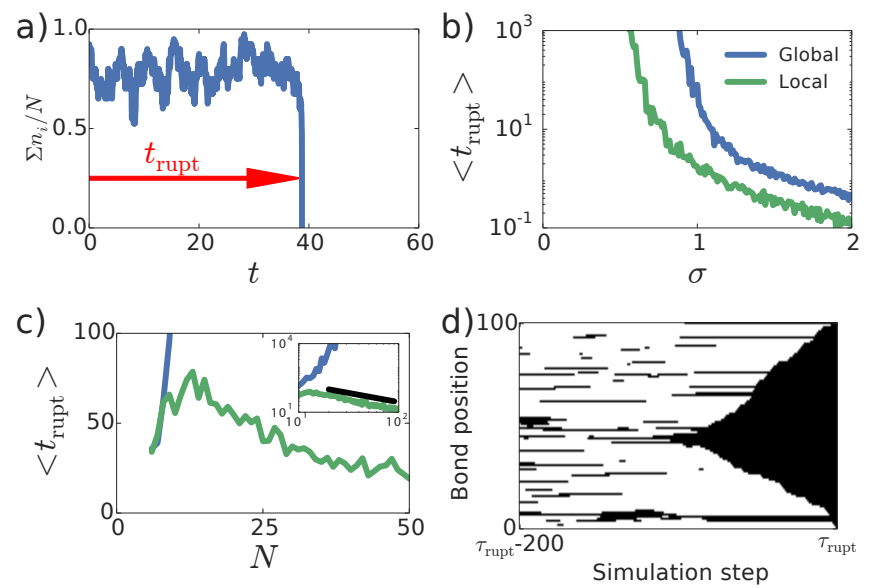

FIG. 2. Stochastic rupture of simulated 1D transient networks subject to a mechanical stress. a) Typical example of the fraction of closed bonds in time upon application of stress, after $t=t_{\text {rupt }}$, spontaneous fracture occurs ( $K=0.9, N=20, \sigma=0.7$, global load sharing). b) Stress dependence of rupture time. Although quantitatively different, global and local load sharing show qualitatively similar behavior with two exponential regimes with a cross over at around $\left\langle t_{\text {rupt }}>\approx 1(K=0.9, N=20)\right.$. c) The system size dependence of the rupture time reveals a qualitative difference between global and local load sharing: whereas the rupture time increases with system size for global load sharing, local load sharing shows an optimum in strength at a well-defined system size $(K=0.9, \sigma=0.7)$. Inset: same data on a log-log scale, showing that after a critical system size $<t_{\text {rupt }}>\sim N^{-1}$ for local load sharing. d) Kymograph of crack initiation under local load sharing (white $=$ closed, black=open). Plotting the bond state as a function of position (y-axis) versus simulation step ( $\mathrm{x}$-axis) clearly reveals how bond opening proceeds via a well-defined crack. The $\mathrm{x}$-axis shows simulation step rather than time, as the crack propagation is orders of magnitude faster than the crack initiation (figure 2 ).

the system is large compared to the number of ablated bonds, yet small enough to allow for equilibration without spontaneous crack initiaton. Figure 3 a shows how fracturing becomes more likely upon increasing the ablation size $l_{\text {ablate }}$, and that the required ablation size $l_{\text {ablate }}$ to initiate fracturing decreases with the applied stress $\sigma$. Figure $3 \mathrm{~b}$ shows that an increase of bond affinity $K$ increases the critical ablation size $l_{\text {ablate }}$ required for triggering fracture.

To quantitatively understand the ablation data, we define crack length $L$ as the largest bond distance $l_{i}$ in the system. In case of global load sharing, the force on bonds at the edge of the crack stays independent of $L$ as long as $\mathrm{L} \ll \mathrm{N}$, so ablation does not induce fracture. By contrast, in case of local load sharing, the force on the bond at the edge of the crack is $f=\sigma \cdot L \cdot 1 / 2$ (the factor $1 / 2$ is because the load on the hole is shared by the bonds at both ends). Thus, $k_{\text {off }}$ exponentially grows with the crack size due to a linearly increasing force, whereas the chance of rebinding increases only linearly due to a larger area in which rebinding can occur. As a result, for large enough $l_{\text {ablate, }}$, bond unzipping will occur for any system under stress.

We are interested in the length $L_{\text {unstable }}$ at which the crack becomes unstable. As a first order approximation, we can find the fixed points of crack length $\stackrel{*}{L}$ by calculating the length at which the rates of bond opening and closing are equal (figure 4 a):

$$
2 \cdot k_{\mathrm{off}}(1 / 2 \cdot \sigma \cdot \stackrel{*}{L}) \approx k_{\mathrm{on}} \cdot \stackrel{*}{L}
$$

This condition is met at the average bond distance at equilibrium, $L_{\text {stable }}$, and the bond distance at the unstable point, $L_{\text {unstable: }}$ :

$$
\begin{gathered}
L_{\text {stable }}(\sigma, K) \approx 2 \cdot \frac{W_{0}\left(-\sigma \cdot\left(\frac{1}{K}-1\right)\right)}{-\sigma} \\
L_{\text {unstable }}(\sigma, K) \approx 2 \cdot \frac{W_{-1}\left(-\sigma \cdot\left(\frac{1}{K}-1\right)\right)}{-\sigma}
\end{gathered}
$$

where $W$ is the Lambert $\mathrm{W}$ function with $W_{0}$ the main branch and $W_{-1}$ the second branch [36]. Note that the network transitions from metastable to unstable at $L_{\text {stable }}=L_{\text {unstable }}$ (seen as a change in slope in figure $2 \mathrm{p}$ at around $\left\langle t_{\text {rupt }}>\approx 1\right.$ ). For local load sharing, the transition from a metastable to an unstable network occurs beyond a critical bond-to-bond distance $L_{\text {unstable, whereas }}$ for global load sharing this transition occurs beyond a critical fraction of open bonds and therefore explains the continuous increase of rupture time as function of time [9, 17-19].

To test equation 7, we show in figure 4b that all ablation data can be successfully collapsed onto a single master curve using a normalized ablation size $\frac{l_{\text {ablate }}}{L_{\text {unstable }}}$. To compare equation 7 with both the ablation data and the typical length scale observed in figure $2 \mathrm{~b}$, we first define a critical ablation length, $l_{\text {crit }}$, which we obtain by fitting the size dependence of the rupture probability $\phi_{\text {rupt }}$ to a sigmoidal function $\phi_{\text {rupt }}=\frac{1}{1+e^{-l} \text { crit }-l_{\text {ablate }}}$ at each applied stress and at bond affinity $K=0.9$. We can now combine the critical ablation length $l_{\text {crit }}$ from figure $3 \mathrm{a}$ with the optimal system size $N$ found in figure $2 \mathrm{k}$ and conclude that all these data are well-described by equation 7 (figure 44).

Up to now, theoretical work on transient network fracturing has been limited to the assumption of global load sharing [9, 17 19$]$. We find that $\left\langle t_{\text {rupt }}>\right.$ is insensitive to this assumption for microscopic systems (up to approximately 10-100 bonds, see equation 7 and figure 22). However, fracturing of larger system follows fundamentally different rules, in which the notion of local load sharing becomes important. Our study investigates the idealized limit of fully localized load sharing, but we observe similar behavior of a typical fracture length when the load distribution is simulated via a mechanical model [35.

Our model predicts features which are different from global load sharing, but consistent with experimental observations on a wide range of viscoelastic materials. First, 

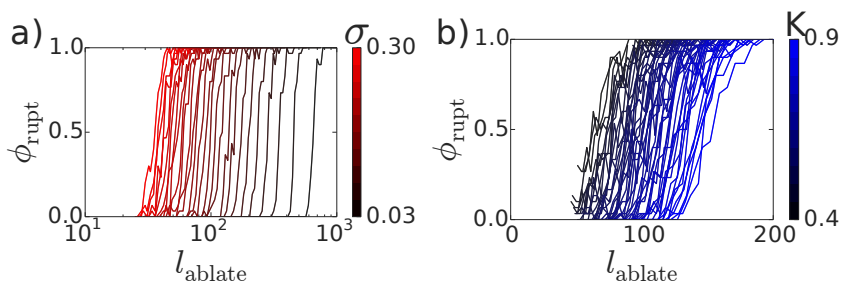

FIG. 3. Characterization of critical crack length in local load sharing Ablation experiments were performed by first equilibrating the system under stress until $t=1$, next bonds were ablated: $n_{i}=0$ for $\mathrm{i}=0 \ldots l_{\text {ablate }}$. After the ablation, the network was studied up to $t=2$. This experiment was repeated 30x per condition, and the fraction of observed ruptures $\phi_{\text {rupt }}$ was recorded. We plot the ablation size $l_{\text {ablate }}$ versus the fraction of observed ruptures $\phi_{\text {rupt }}$ for different values of a) applied stress $\sigma$ at $K=0.9$ or b) bond affinity $K$ at $\sigma=0.1$. Control ablation experiments using the same parameter with global load sharing never showed fracturing.
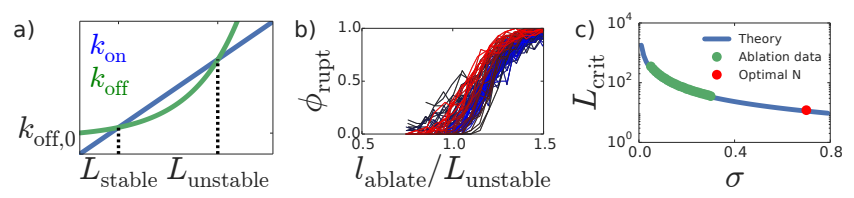

FIG. 4. Comparison between theory and simulation a) As a function of crack size, the on-rate increases linearly, whereas the off-rate increase exponentially. As a result, the crack becomes unstable after $L_{\text {unstable }}$. b) All data from figure 3 can be collapsed obtained onto a single master curve by normalizing according to $l_{\text {ablate }} /\left(L_{\text {unstable }}(\sigma, K)\right)$. c) Equation 7 quantitatively predicts both the critical length for ablation, and the width at which the maximal rupture time is observed in figure 2 .

rupturing of macroscopic viscoelastic materials proceeds via spontaneous crack initiation, different from the homogeneous failure predicted by global load sharing models [9, 17-19]. This prediction is borne out by experimental observations of a wide range of viscoelastic materials [3, 11, 31, 32]. Second, the model of global load sharing predicts that the rupture time strongly increases with the system size. As a result, delayed fracturing $\left(<t_{\text {rupt }}>\gg k_{\text {on }}\right)$ would only be experimentally observable very close to the critical stress for any macroscopic system. Instead, delayed fracture is experimentally observed for many different viscoelastic materials over a wide range of stresses [3, 8-10]. We find that in case of local load sharing, the dependence of $\left\langle t_{\text {rupt }}>\right.$ on $\sigma$ does not diverge upon increasing system size $N$. Thus, our model for the first time explains why delayed fracturing is readily observable on laboratory timescales over a wide range of stresses in experiments.

The model makes several concrete predictions that can be tested experimentally by applying shear stress on viscoelastic materials. Firstly, we predict that the average rupture time measured at constant stress will be inversely proportional to the system size (figure 2p) as the crack initiation rate is constant per volume. Secondly, the presence of a critical crack length can be measured directly by performing laser ablation on viscoelastic materials under stress, a technique that is common in biophysical studies of cell and tissue tension [37. Thirdly, the dependence of the critical crack length on the applied stress and bond kinetics (Equation 7) can be tested experimentally. The bond kinetics can for instance be experimentally controlled by changing the temperature in cross linked actin networks 38 or salt conditions in polyelectrolyte gels [39].

Our framework for understanding the crack initiation process in viscoelastic materials can be used to rationally design more robust materials. We have considered evenly distributed bonds. For future work, it would be interesting to investigate the effect of inhomogeneity under local load sharing. It is interesting to note that cellular adhesion proteins are not randomly distributed but clustered with a well-defined size 40. Simulations have shown that an intermediate degree of clustering is optimal for preventing fracturing [41, 42, although the nature of this optimum remained poorly understood. We speculate that this optimal clustering density is related to the critical length scale for crack initiation and that this strategy of clustering bonds is an interesting design principle for synthetic materials.

\section{ACKNOWLEDGEMENTS}

We thank Pieter Rein ten Wolde, Chase Broedersz, David Brueckner and Mareike Berger for fruitful discussions. This work is part of the research program of the Netherlands Organisation for Scientific Research (NWO). We gratefully acknowledge financial support from an ERC Starting Grant (335672-MINICELL).
[1] Chase P. Broedersz, Martin Depken, Norman Y. Yao, Martin R. Pollak, David a. Weitz, and Frederick C. MacKintosh. Cross-link-governed dynamics of biopolymer networks. Physical Review Letters, 105(December):1-4, 2010.

[2] Ecaterina Stela Dragan. Design and applications of interpenetrating polymer network hydrogels. A review. Chem- ical Engineering Journal, 243:572-590, 2014.

[3] Paulina J. Skrzeszewska, Joris Sprakel, Frits a. de Wolf, Remco Fokkink, Martien a. Cohen Stuart, and Jasper van der Gucht. Fracture and Self-Healing in a WellDefined Self-Assembled Polymer Network. Macromolecules, 43(7):3542-3548, 2010. 
[4] Peter J. Lu and David A. Weitz. Colloidal Particles: Crystals, Glasses, and Gels. Annual Review of Condensed Matter Physics, 4(1):217-233, 2013.

[5] Fanlong Meng, Robyn H. Pritchard, and Eugene M. Terentjev. Stress Relaxation, Dynamics, and Plasticity of Transient Polymer Networks. Macromolecules, 49(7):2843-2852, 2016.

[6] Lars Wolff, Pablo Fernandez, and Klaus Kroy. Resolving the Stiffening-Softening Paradox in Cell Mechanics. PLoS ONE, 7(7):e40063, 2012.

[7] Hyun Joon Kong, Emma Wong, and David J. Mooney. Independent control of rigidity and toughness of polymeric hydrogels. Macromolecules, 36(12):4582-4588, 2003.

[8] Thomas Gibaud, Frelat Damien, and Sébastien Manneville. Heterogeneous yielding dynamics of a colloidal gel. Soft Matter, 6:3482-3488, 2010.

[9] Joris Sprakel, Stefan B. Lindström, Thomas E. Kodger, and David A. Weitz. Stress enhancement in the delayed yielding of colloidal gels. Physical Review Letters, 106(24):1-4, 2011.

[10] J F Berret and Y Séréro. Evidence of shear-induced fluid fracture in telechelic polymer networks. Physical Review Letters, 87:048303, 2001.

[11] H. Tabuteau, S. Mora, G. Porte, M. Abkarian, and C. Ligoure. Microscopic mechanisms of the brittleness of viscoelastic fluids. Physical Review Letters, 102(15):14, 2009.

[12] José Alvarado, Michael Sheinman, Abhinav Sharma, Fred C. MacKintosh, and Gijsje H. Koenderink. Molecular motors robustly drive active gels to a critically connected state. Nature Physics, 9(9):591-597, aug 2013.

[13] J. Sprakel, E. Spruijt, J. van der Gucht, J.T. Padding, and W.J. Briels. Failure-mode transition in transient polymer networks with particle-based simulations. Soft Matter, 5(23):4748, 2009.

[14] Alessandro Lucantonio, Giovanni Noselli, Xavier Trepat, Antonio Desimone, and Marino Arroyo. Hydraulic Fracture and Toughening of a Brittle Layer Bonded to a Hydrogel. Physical Review Letters, 115(18):1-5, 2015.

[15] A.A. Griffith; and M Eng. VI. The phenomena of rupture and flow in solids. 1920.

[16] Giovanni Nava, Marina Rossi, Silvia Biffi, Francesco Sciortino, and Tommaso Bellini. Fluctuating Elasticity Mode in Transient Molecular Networks. Physical Review Letters, 119(7):1-5, 2017.

[17] G I Bell. Models for the specific adhesion of cells to cells. Science (New York, N.Y.), 200(4342):618-627, 1978.

[18] U Seifert. Rupture of multiple parallel molecular bonds under dynamic loading. Physical review letters, 84(12):2750-2753, 2000.

[19] T. Erdmann and U. S. Schwarz. Stability of Adhesion Clusters under Constant Force. Physical Review Letters, 92(10):108102-1, 2004.

[20] Hendrik Dietz and Matthias Rief. Elastic bond network model for protein unfolding mechanics. Physical Review Letters, 100(9):1-4, 2008.

[21] D. A. Head, A. J. Levine, and F. C. MacKintosh. Distinct regimes of elastic response and deformation modes of cross-linked cytoskeletal and semiflexible polymer networks. Physical review. E, Statistical, nonlinear, and soft matter physics, 68(6):061907, 2003.

[22] Jader Colombo, Asaph Widmer-Cooper, and Emanuela Del Gado. Microscopic picture of cooperative processes in restructuring gel networks. Physical Review Letters, 110(19):1-5, 2013.

[23] Anindita Basu, Qi Wen, Xiaoming Mao, T. C. Lubensky, Paul A. Janmey, and A. G. Yodh. Nonaffine displacements in flexible polymer networks. Macromolecules, 44(6):1671-1679, 2011.

[24] J. Liu, G. H. Koenderink, K. E. Kasza, F. C. MacKintosh, and D. A. Weitz. Visualizing the strain field in semiflexible polymer networks: Strain fluctuations and nonlinear rheology of F-actin gels. Physical Review Letters, 98(19):1-4, 2007.

[25] Qi Wen, Anindita Basu, Paul a. Janmey, and Arjun G. Yodh. Non-affine deformations in polymer hydrogels. Soft Matter, 8(31):8039-8049, 2012.

[26] T. S. Majmudar and R. P. Behringer. Contact force measurements and stress-induced anisotropy in granular materials. Nature, 435(7045):1079-1082, 2005.

[27] Jun Guo, Yuexiu Wang, Frederick Sachs, and Fanjie Meng. Actin stress in cell reprogramming. Proceedings of the National Academy of Sciences, 111(49):E5252-E5261, 2014.

[28] Richard C. Arevalo, Pramukta Kumar, Jeffrey S. Urbach, and Daniel L. Blair. Stress heterogeneities in sheared type-i collagen networks revealed by boundary stress microscopy. PLoS ONE, 10(3):1-12, 2015.

[29] Christian Vaca, Roie Shlomovitz, Yali Yang, Megan T. Valentine, and Alex J. Levine. Bond breaking dynamics in semiflexible networks under load. Soft Matter, 11(24):4899-4911, 2015.

[30] Matti Gralka and Klaus Kroy. Inelastic mechanics: A unifying principle in biomechanics. Biochimica et Biophysica Acta (BBA) - Molecular Cell Research, 2015.

[31] Joseph R. Gladden and Andrew Belmonte. Motion of a viscoelastic micellar fluid around a cylinder: Flow and fracture. Physical Review Letters, 98(22):1-4, 2007.

[32] Guillaume Foyart, Christian Ligoure, Serge Mora, and Laurence Ramos. Rearrangement Zone around a Crack Tip in a Double Self-Assembled Transient Network. ACS Macro Letters, 5(10):1080-1083, 2016.

[33] Elizaveta a. Novikova and Cornelis Storm. Contractile fibers and catch-bond clusters: A biological force sensor? Biophysical Journal, 105(6):1336-1345, 2013.

[34] Daniel T. Gillespie. A general method for numerically simulating the stochastic time evolution of coupled chemical reactions. Journal of Computational Physics, 22(4):403-434, 1976.

[35] See Supplemental Material.

[36] JH Lambert. Observationes variae in mathesin puram. Acta Helvetica, 1758.

[37] Jean-Yves Tinevez, Ulrike Schulze, Guillaume Salbreux, Julia Roensch, Jean-François Joanny, and Ewa Paluch. Role of cortical tension in bleb growth. Proceedings of the National Academy of Sciences of the United States of America, 106(44):18581-6, nov 2009.

[38] Sabine M. Volkmer Ward, Astrid Weins, Martin R. Pollak, and David A. Weitz. Dynamic Viscoelasticity of Actin Cross-Linked with Wild-Type and Disease-Causing Mutant $\alpha$-Actinin-4. Biophysical Journal, 95(10):49154923, 2008.

[39] Evan Spruijt, Joris Sprakel, Marc Lemmers, Martien A Cohen Stuart, and Jasper Van Der Gucht. Relaxation dynamics at different time scales in electrostatic complexes: Time-salt superposition. Physical Review Letters, 105(20):1-4, 2010. 
[40] Ulrich S. Schwarz and Samuel a. Safran. Physics of adherent cells. Reviews of Modern Physics, 85(3):1327-1381, aug 2013.

[41] Yuan Lin and L. B. Freund. Optimum size of a molecular bond cluster in adhesion. Physical Review E - Statistical, Nonlinear, and Soft Matter Physics, 78(2):1-6, 2008.

[42] Jin Qian, Jizeng Wang, and Huajian Gao. Lifetime and strength of adhesive molecular bond clusters between elastic media. Langmuir, 24(4):1262-1270, 2008.

[43] Robert D. Cook, David S. Malkus, Michael E. Plesha, and Robert J. Witt. Concepts and Applications of Finite Element Analysis. John Wiley 85 Sons, 2007.

\section{Supplementary Information}

To validate the behavior of cracks in viscoelastic materials obtained with the local load sharing assumption, we implemented a 2D mechanical model (figure $\mathrm{S} 5$ ) based on Finite Element Analysis (FEA) 43. The transient bonds, modeled by a linear elastic material with a Young's modulus $E$ and Poisson's ratio $\nu$, are modeled as elastic bodies fixed at their bottom and attached to an upper body with the same material properties. Both the bonds and the elastic body are discretized using bilinear square elements consisting of four nodes. The elastic body consists of $h \cdot N$ elements, where $h$ is the height of the elastic body expressed in terms of the in number of elements and $N$ is the number of bonds. Periodic boundary conditions are applied to the left and right boundaries. To apply tension to the bonds, a vertical displacement is applied to the upper boundary of the solid part, until a force $\sigma_{\mathrm{FEA}}$ is reached. A Monte Carlo scheme similar to the one used for the local load sharing model is applied to determine the transient behavior of the bonds, using equation 2 and 3 from the main text, and we define the stress intensity factor value $\alpha_{i}$ for FEA as:

$$
\alpha_{i}=\frac{U_{i}}{\sum_{i} U_{i}} N
$$

where $U_{i}$ is the bonds' elastic strain energy density which can be found by integrating the stress vector on the bond $\{s\}_{i}$ for the strain vector $\{\boldsymbol{e}\}_{i}$ according to:

$$
U_{i}=\int\{\boldsymbol{s}\}_{i}^{T}\{d \boldsymbol{e}\}_{i}=\frac{1}{2}\left[\begin{array}{lll}
s_{x x} & s_{y y} & s_{x y}
\end{array}\right]_{i}\left[\begin{array}{c}
e_{x x} \\
e_{y y} \\
e_{x y}
\end{array}\right]_{i}
$$

Figure $\$ 6$ shows how the elastic body redistributes the applied force on the bonds. Importantly, we observe a stress intensity distribution for FEA that is comparable to that of the local load sharing assumption using the settings of $h=N$ and $E_{\text {body }}=E_{\text {bond }}$. For future work it will be interesting to vary $h$ and/or $E_{\text {bond }} / E_{\text {body }}$ and test the effect on the stress distribution and subsequent fracturing behavior. 

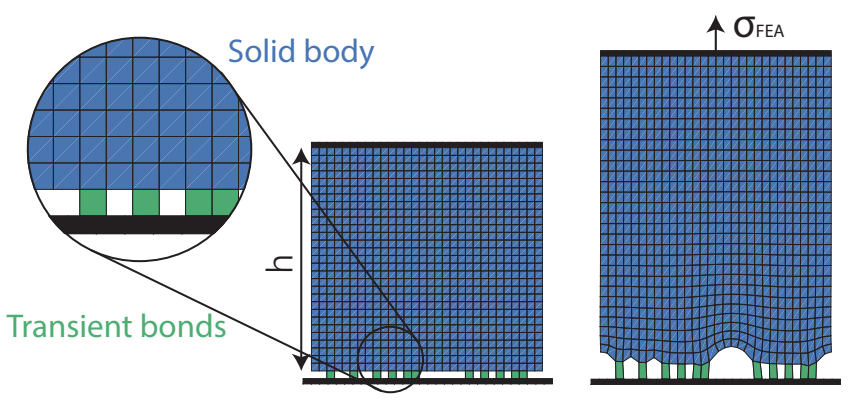

FIG. 5. Finite element model Finite element model representation of an elastic body (blue) of total thickness $h$ with random distribution of bonds (green) at rest (left) and stretched (right). The deformation, due to the applied force $\sigma_{\mathrm{FEA}}$ on the elastic body, is exaggerated for clarity.

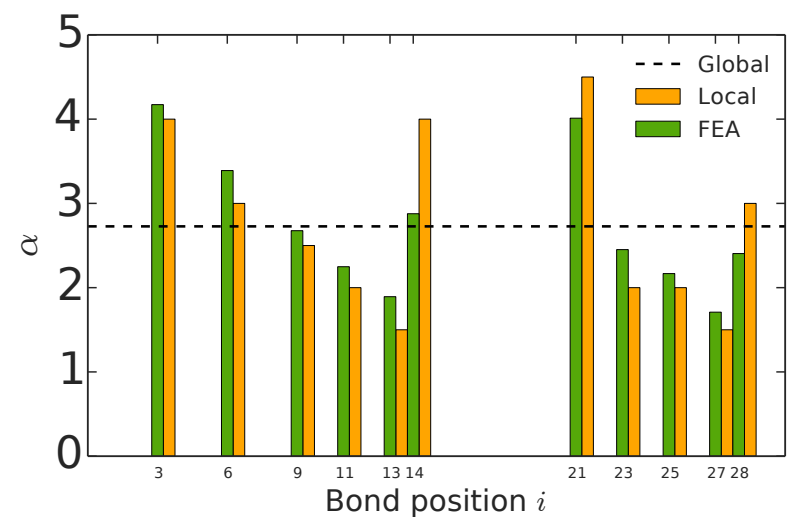

FIG. 6. Comparison of load distribution Stress intensity factor comparison of different modes of load sharing (global, local, FEA) for the bond distribution shown in Figure $\$ 5$.

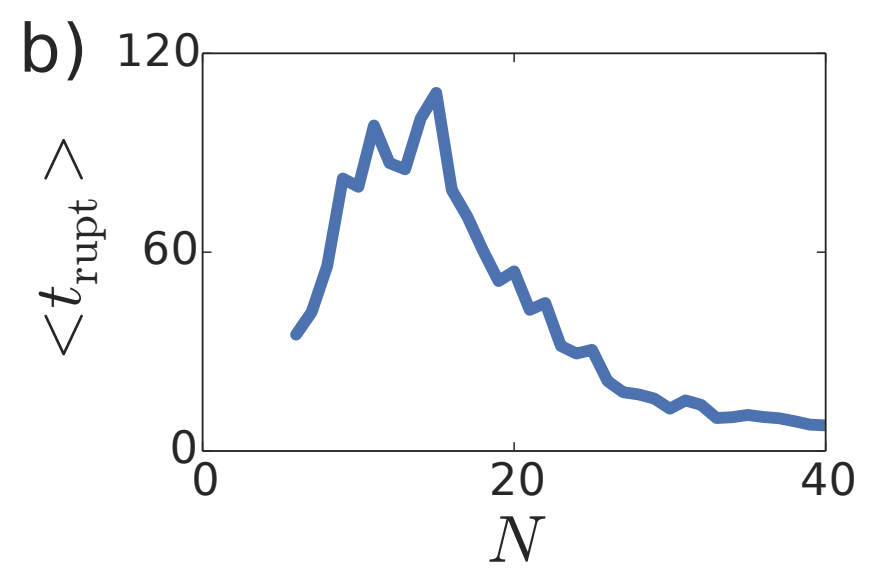

FIG. 7. FEA verifies main qualitative difference between local and global load sharing Unlike global load sharing but similar to local load sharing (main text figure 2c), the average rupture time in FEA is peaked for intermediate system size $N$. 


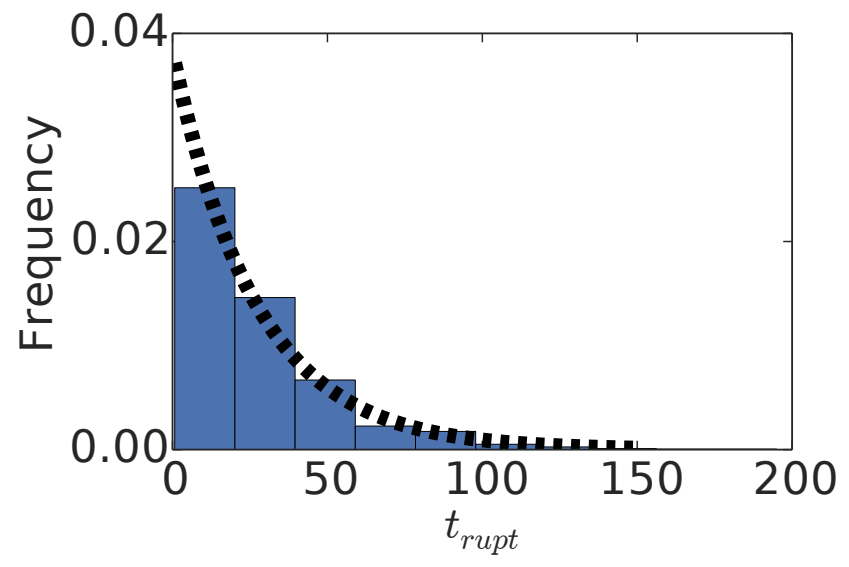

FIG. 8. Rupture times are exponentially distributed We performed fracturing simulations under local load sharing for $1 \mathrm{D}$ networks (see main text figure 1) using identical parameters ( $\sigma=0.7, K=0.9, N=20,1000$ repeats) and recorded $\tau_{\text {rupt }}$. The distribution of rupture times is exponential, suggesting a stochastic process. 